\title{
VALUES CRISIS IN CONTEMPORARY ARCHITECTURE WITH CONCENTRATION ON EGYPTIAN ARCHITECTURE IN ASSIUT CITY AS A MODEL
}

\author{
Ahmed Helal Mohammed and \\ Professor of Architectural designs, King Abdeh Aziz University, \\ Jeddeh, POB 8020 Jeddeh 21589,Email:dra_helal@yahoo.com.
}

\section{Amar Sadeq Dahlan}

Head of the Architecture department, Faculty of Environmental Designs, Jeddeh,POB 8020 Jeddeh 21589

\section{(Received July 5, 2008 Accepted August 11, 2008)}

It is noted that the contemporary architecture has influenced by western architectural styles and it has just become an imitation to west models which reflect western values and traditions that are different from Islamic values, thus contemporary architecture lacked all human and social values. The study aims at studying values in contemporary architecture to check the values that must be developed to get architectural model that suits the needs and requirements of the inhabitants and their Islamic instructions. To achieve these aims, the researcher determined some pivots by which he can achieve these goals. The researcher concluded the required values that must be prevailing in architecture: achieving privacy by its types, caring for family, neighbors' rights and the social values. The study revealed that the factors that lead to lack values and their deterioration in contemporary architecture are: the deterioration of Islamic civilization, the education of the architect, the deterioration of economic level, laws of building, mass media and financial factors. The study revealed that the contemporary architecture lacked human and social values which were greatly prevailing in past architecture. At the end, the study concluded that contemporary architecture in Assiut city lacked the values whether in governmental, official or random architecture. 


\section{أزمة القيم في العمارة المعاصرة - مع التركيز على العمارة المصرية المعاصرة في مدينة

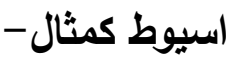 \\ أعداد/ا. د.احمد هلال محمد1 د / عمار صادق دحلان2 2}

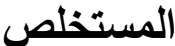

إن ضياع القيم في العمارة المعاصرة يعود إلى العديد من الأسباب ونقصد بـالقيم هنـا

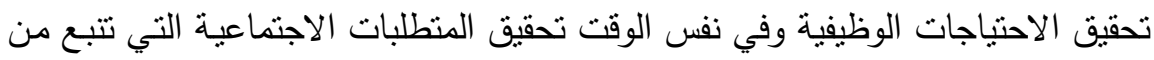
العقيدة الإسـلامية التي لا ينفصل فيها البناء عن الأسرة التي تقيم فيه.والمتأمل للعمارة

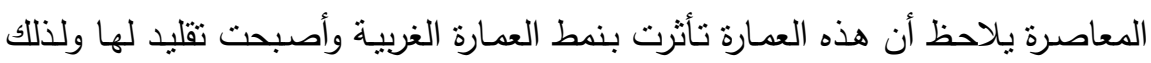
عبرت هذه العمارة عن تقاليد وعادات مجتمعات غربية بعيدة عن قيمنا ومبادئنا المستمدة من الثريعة الإسلامية وأصبحت فاقدة لكل القيم الإنسانية والاجتماعية.

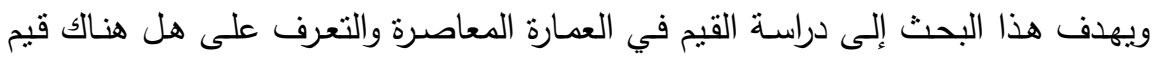

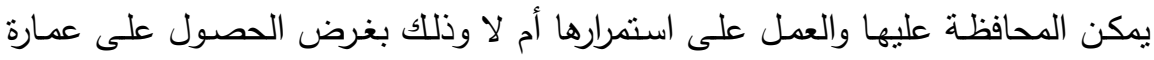

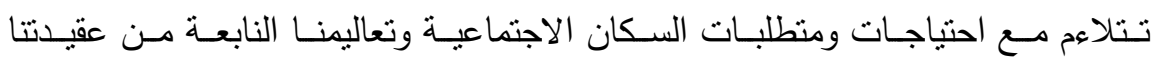
الإسلامية ـ ولتحقيق هذه الأهداف فانه تم تحديد عدد من المحاور المحددة لهذا البحث والتي أمكن من خلال دراستها الوصول إلى تحقيق هذه الأهداف.

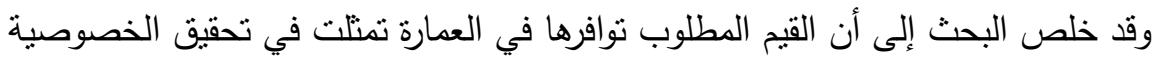

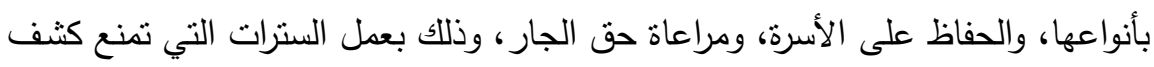

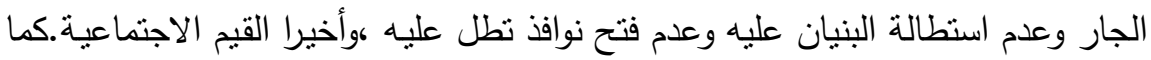

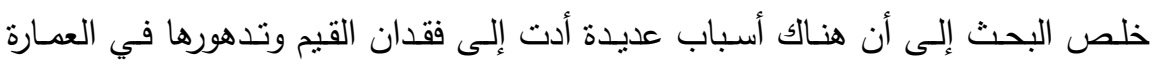

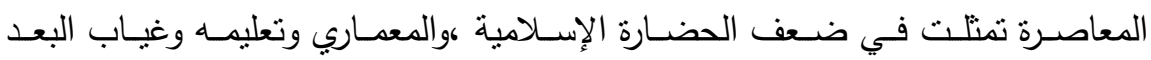

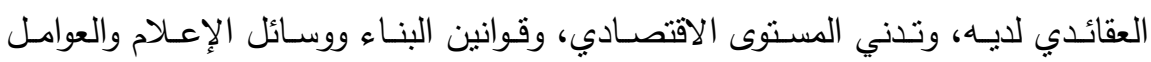

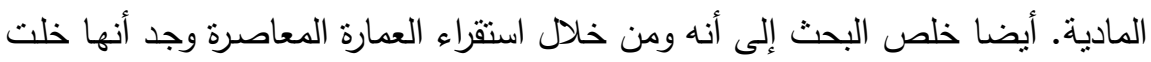
من القيم الإنسانية والاجتماعية التي كانت تصمم عليها وقد ظهر ذلك واضحا في انفتاح

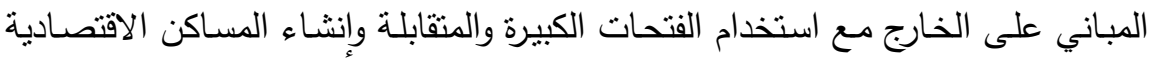

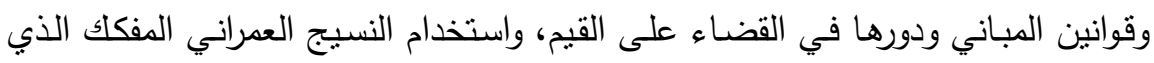
أدى إلى العزلة الاجتماعية وضعف الروابط وليط بين السكان. ومن خلال دراسة الحالة التي التي

1 ـ أستاذ التصميم المعماري ـ قسم العمارة- كلية تصاميم البيئة - جامعة الملك عبد العزيز. جده ص.ب: 8020 جده

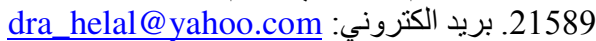
2 - رئيس قسم العمارة - ـ - كلية تصاميم البيئة - جامعة الملك عبد العزيز . جه ص.ب: 8020 جده 21589 
تتاولها البحث خلص إلى أن العمارة المعاصرة في مدينة أسيوط خلت من القيم بل يمكن القول بأنه لا يوجد قيم سواء كانت في العمارة الحكومية أو الرسمية والعشوائية.

\section{مقدمـة}

إن ضباع القيم في العمارة المعاصرة يعود إلى العديد من الاسباب منها أنه لم يعد لصاحب المبنى اي

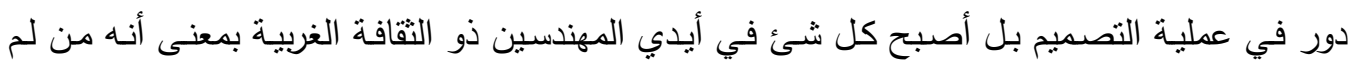

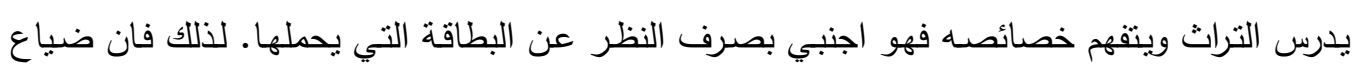

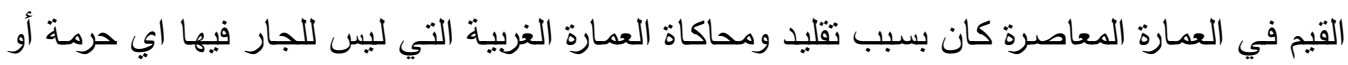
لحرقة الثمس اي اعتبار. والقيم هنا تعني تحقيق الاحتياجات الوظيفية وفي نفس الوقت تحقيق المنطلبات الاجتماعية التي تتبع من

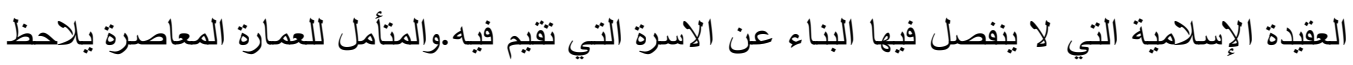

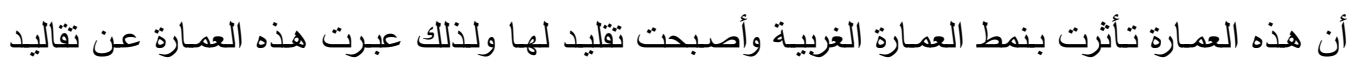

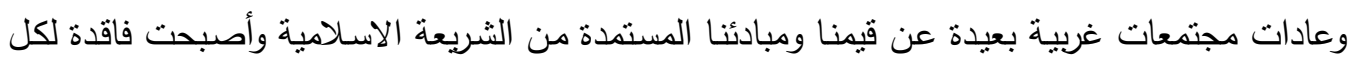

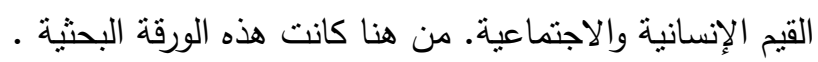

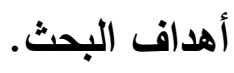

يهدف هذا البحث إلى دراسة القيم في العمارة المعاصرة والتعرف على هل هنالك قيم يمكن المحافظة

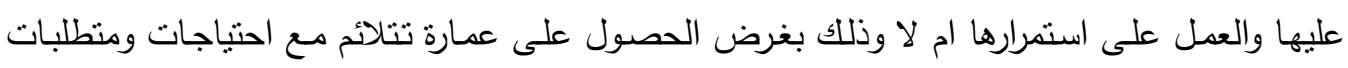

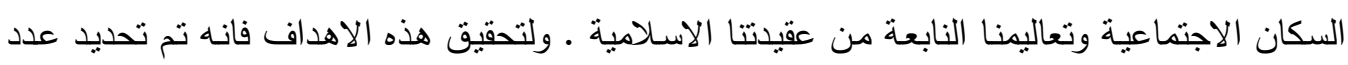
من الحاور المحددة لهذا البحث والتي يمكن من خلال دراستها الوصول إلى تحقيق هذه الأهداف.

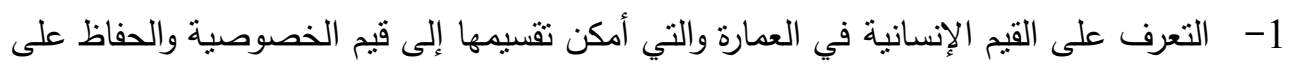

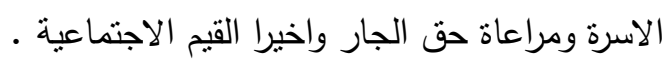
2- - دراسة أسباب تدهور وفقدان القيم في العمارة المعاصرة. 3- - التعرف على مظاهر تدهور القيم في العمارة المعاصرة. 4- دراسة القيم في العمارة المصرية المعاصرة في مدينة أسيوط كمثال.

\section{منهج البحث.}

اعتمدت منهجية البحث لتحقيق الاهداف السابقة على الاتي:-

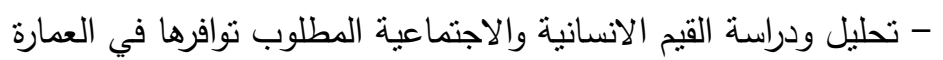
- تحليل اسباب تدهور وفقدان القيم في العمارة ومن ثم التعرف على مظاهر تدهورهاء 
- دراسة حالة العمارة في مدينة اسيوط: اعتمدت هذه الدراسة على تحليل للعمارة وعلى المشاهدة والصور الميدانية والمعايثة الفعلية لهذه الددينة.

\section{1- القيم الانسانية والاجتماعية في العمارة. 1-1-1 تحقيق الخصوصية}

يأتي موضوع تحقيق الخصوصية في اطار المبدأ المعروف لا ضرر ولا ضرار ولذلك اصدر الفقهاء

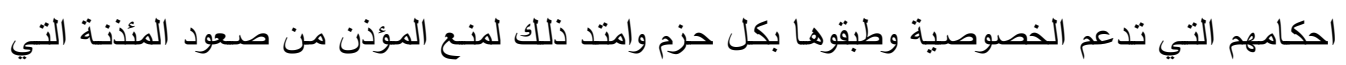

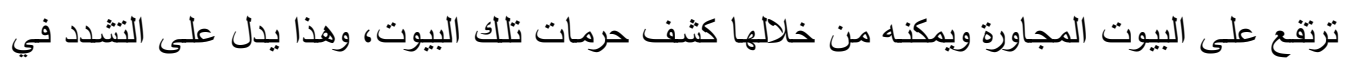

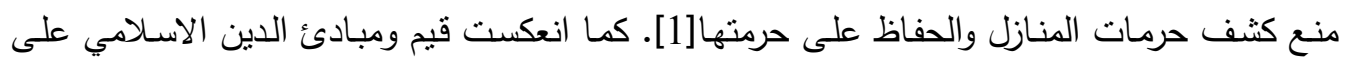

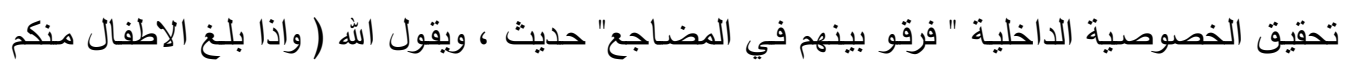

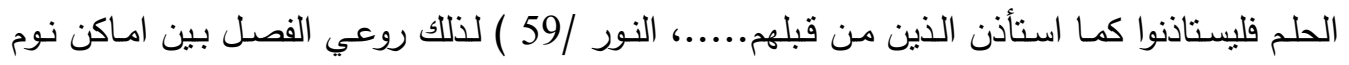

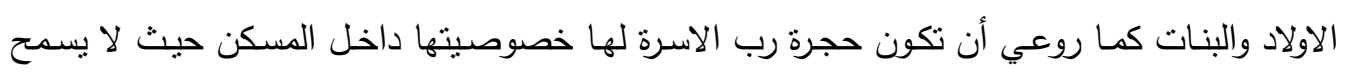

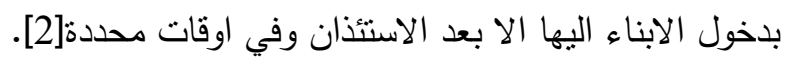

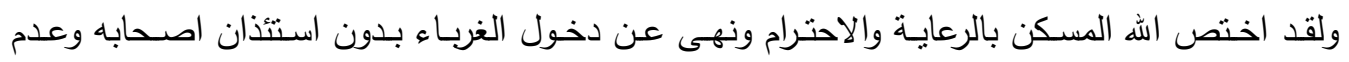

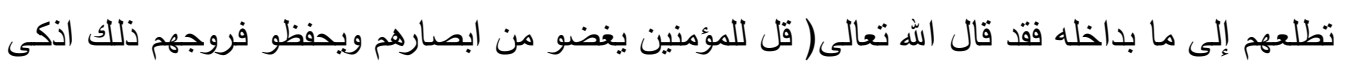
لهم ان اله خبير بما يصنعون , النور /30).

\section{-1 - 2-1 الحفاظ على الاسرة.}

الأسرة هي لبنة المجتمع وقد دعا الاسلام للفصل بين الرجال والنساء وبين الاولاد والبنات ، لذلك فإنه الكه يستوجب توفير الخصوصية بحيث يكون البيت سكنا وآمنا للاسرة ـ وللحفاظ على الاسرة فانه يلزم الاتي[3]:

- توفير الخصوصية لمداخل المساكن. - معالجة الفتحات التي نطل على الخارج.

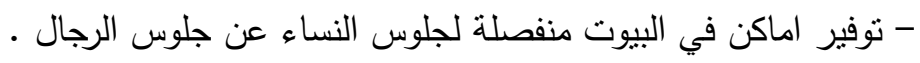

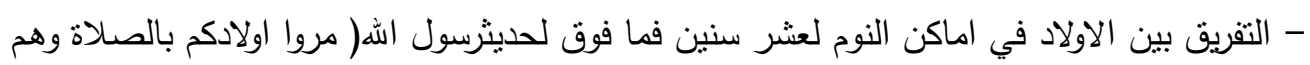
ابناء سبع واضربوهم عليها وهم ابناء عشر سنين وفرقوا بينهم في المضاجع).

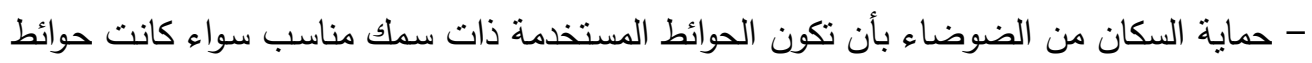
خارجية او داخلية. 1-3- مراعاة حق الجار 
تدعو الاحاديث النبوية إلى احترام الجار ( قال رسول الله مازال جبريل يوصيني بالجار حتى ظننت انه ،

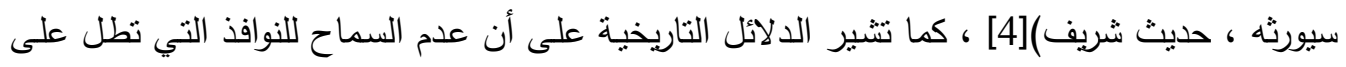

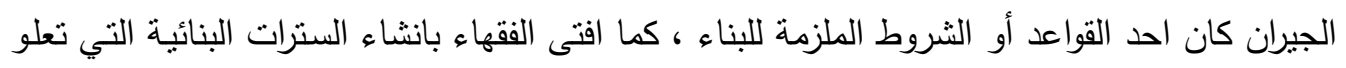

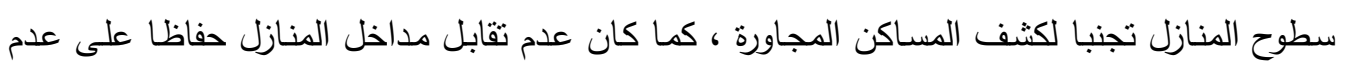
كثف حرمات المساكن المثقابلة[2]. والحفاظ على حقوق الجار تشمل الا يستطيل عليه في البنيان فيسد عليه الهواء إلا باذنه ولا يفتح عليه

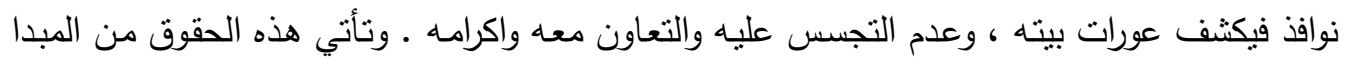

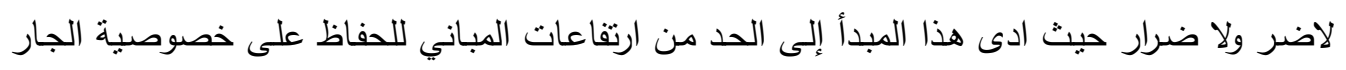

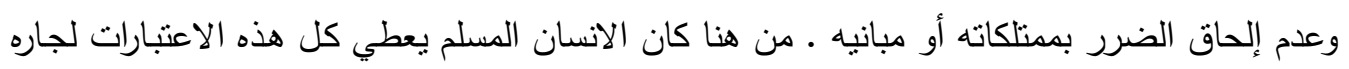
خشية أن يضره أو يجرحه او يصييه باي اذى[5].

\section{-1 - 1-القيم الاجتماعية}

وتتحقق القيم الاجتماعية باستخدام النسيج العمراني الذي يوفر الثقارب والتكامل وتتمية روح الجوار بين

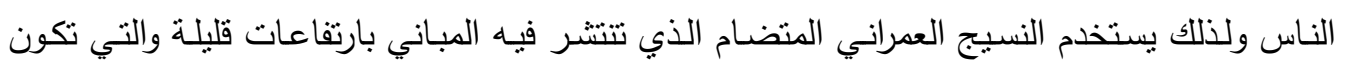

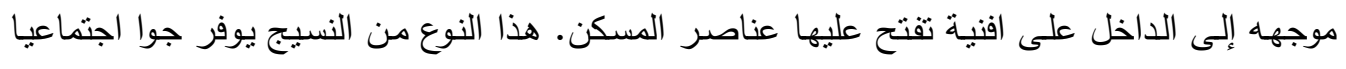

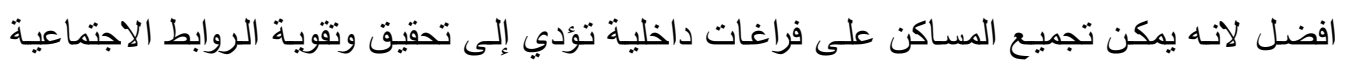

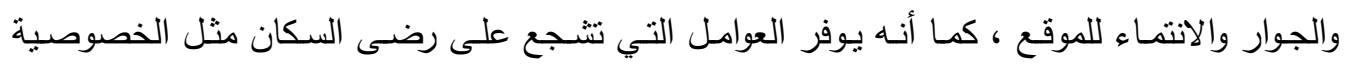

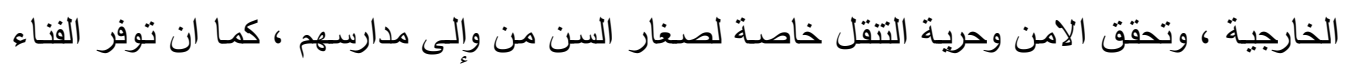

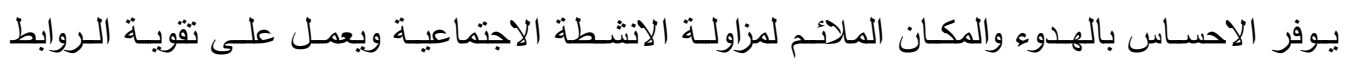

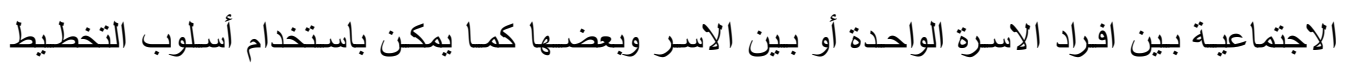

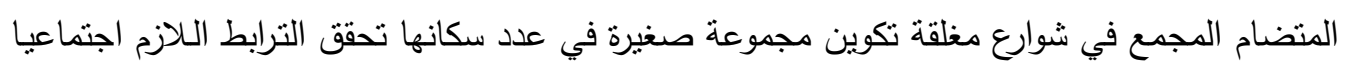

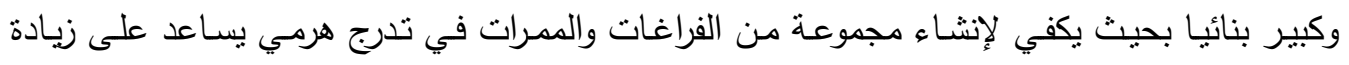
الترابط الاجنماعي المطلوب [6].

2- أسباب تلهور القيم في العمارة المعاصرة.

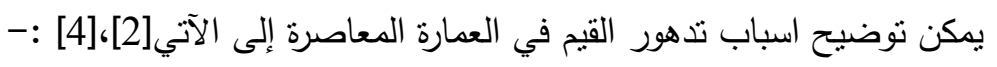

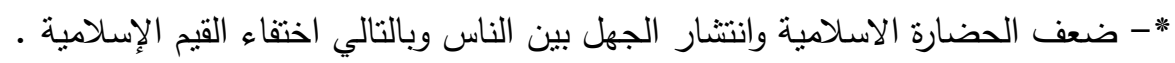

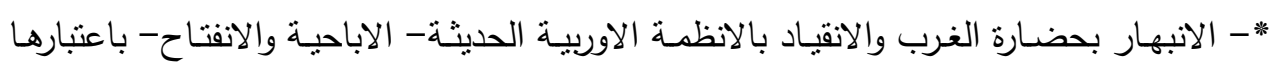
احدى مقومات الحضارة والتطور • 
*- البعثات العلميـة إلى جامعات الغرب أدت إلى ظهور جيل من المعماريين المسلمين بثقافة

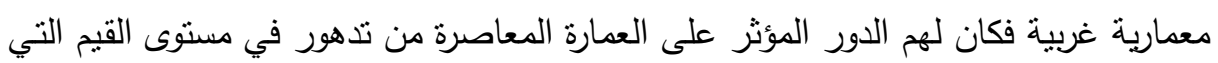
كانت تتحلى بها والذي اثز بدوره على مستوى تعليم العمارة . *- تدني المستوى الاقتصـادي وتداعيه ادى إلى تفكير الفرد يتجهـ فقط نحو ايجـاد مسكن بـاي كيفية دون النظر إلى توفير مبادئ القيم من الخصوصية وغيرها.

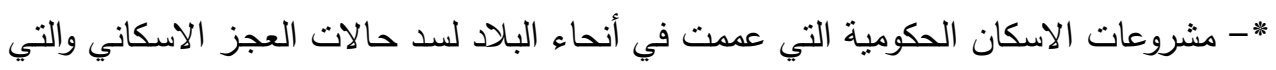

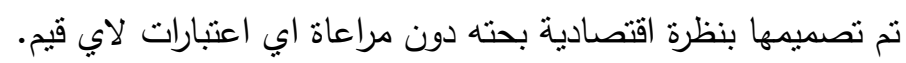

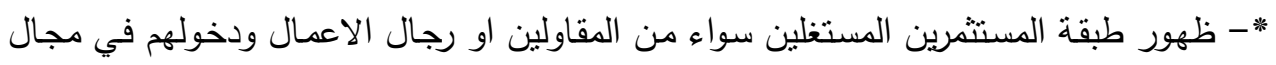

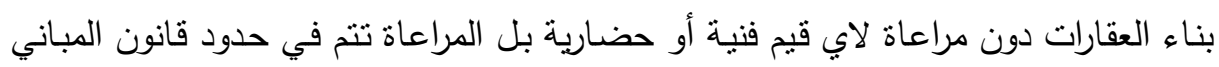
والذي ينم التحايل عليه بطرق مختلفة. * قوانين البناء المستوردة والتي تم صباغتها لتواجه مشكلات الإسكان والزيادة السكانية ساهمت

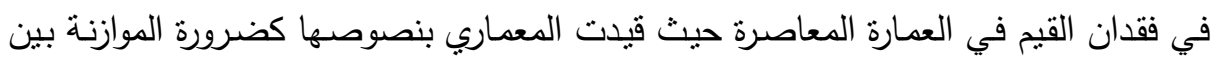

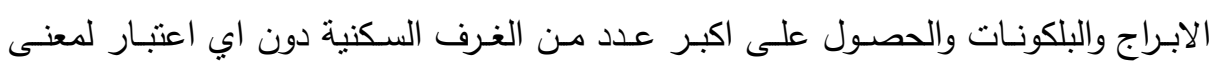
الخصوصية او المعالجات المعمارية التي تحقق ذلك.

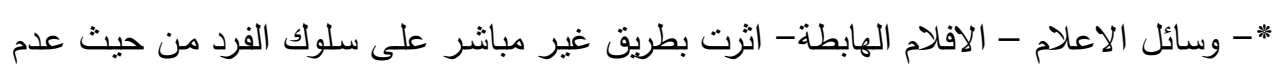
تقيده بالقيم الاسلامية في مسكنه.

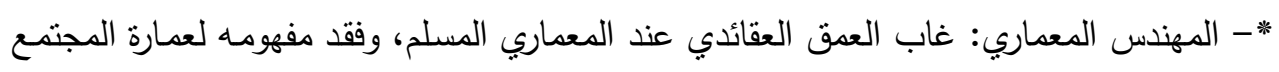

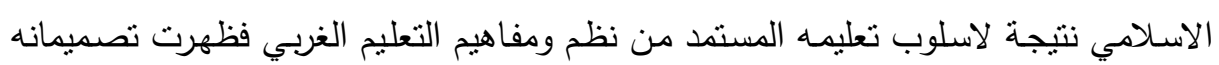
خالية من القيم.

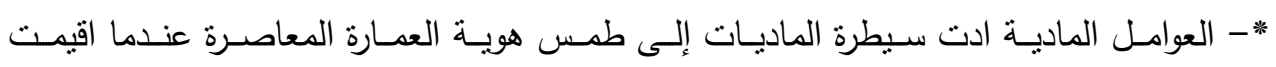

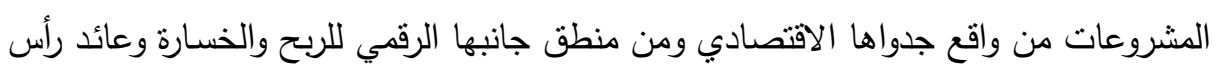

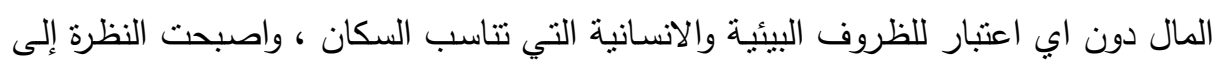

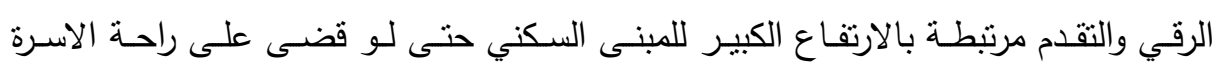
وخصوصيتها.

\section{3- مظاهر تدهور القيم في العمارة المعاصرة.} المتأمل للعمارة المعاصرة يجد أنها خلت من القيم الانسانية والاجتماعية التي كانت تصمم عليها ، ولم

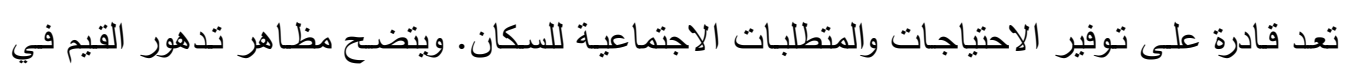

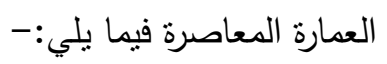


* توجيه العمارة للخارج واستخدام الفتحات الكبيرة والثرفات سـاهم قي القضـاء على الخصوصية الخارجية للمسكن ، كما أنه لا بوجد فصل بين غرف النوم وصالة المعيسة مما ادى إلى فقدان الخصوصية الداخلية.

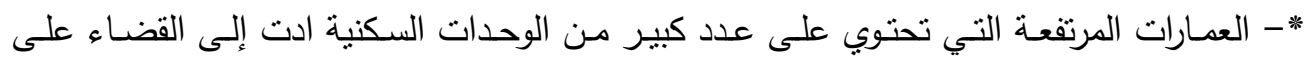

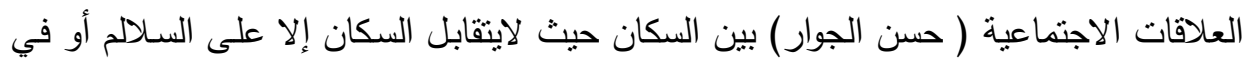
المصاعد واصبح من الصعب على الساكن أن ينعرف على جيرانه الذين يسكنون معه شكل رقم

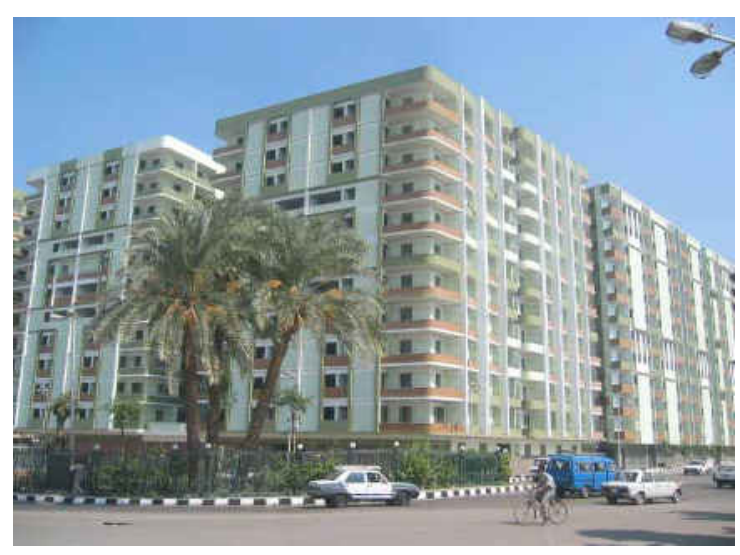

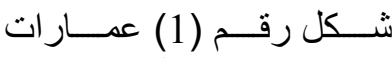
مرتفعة تحنوي على عدد كبير من الوحدات السكنية ادت إلى كئ

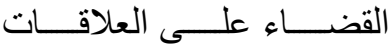
[الاجتماعية

* - فتحات النوافذ الكبيرة والمنقابلة مع ضيق الثوارع واستخدام حوائط بسمك صغير (12سم) اتاح فرصة لاتنقال الضوضاء إلى داخهل المسكن (فقدان الخصوصية السمعية).

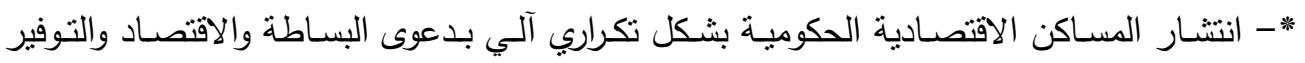

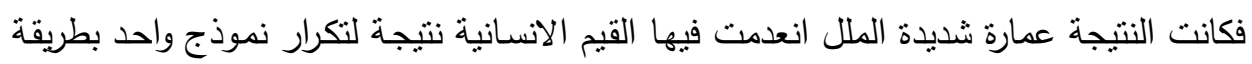
مملة جاءت فيه تصميمات المساكن مفتقرة لكل القيم المطلوبة توفرها في العمارة [7].

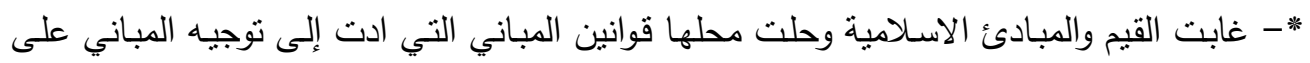

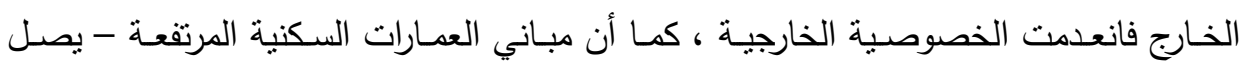
ارتفاعها إلى 36متر - التي تسمح بها قوانين المباني قضت على التى الخصوصبة الخارجيـة لان

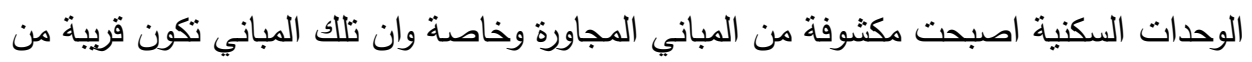
بعضها والفتحات منقابلة شكل رقم(2) [8]، [9]. 


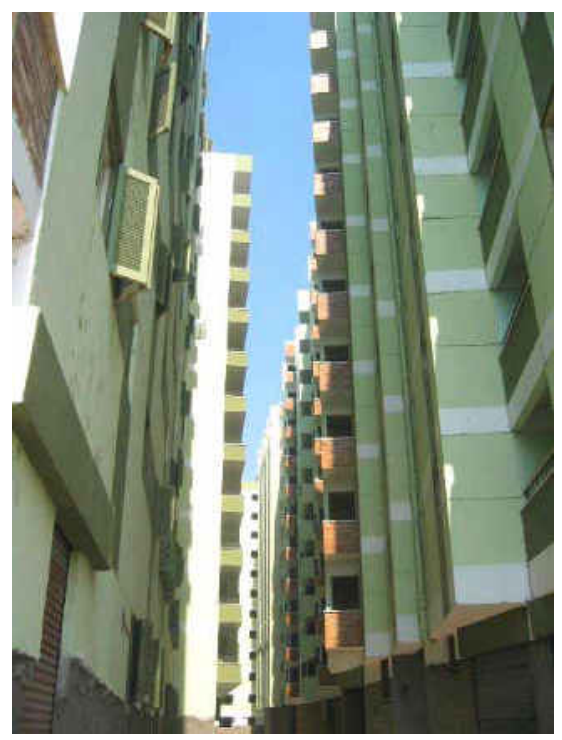

شكل رقـم(2) غابـات الأبـر اج

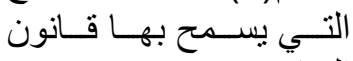

المباني [14]

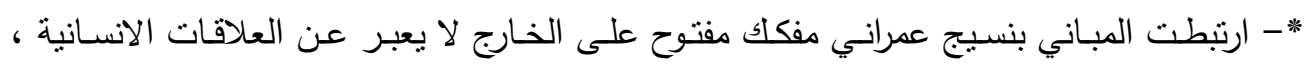

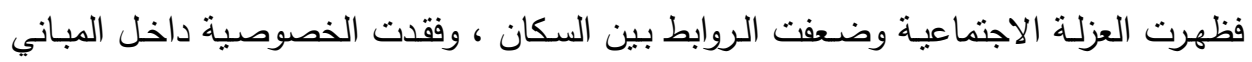

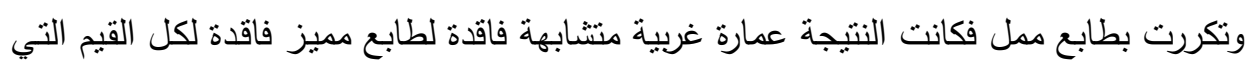

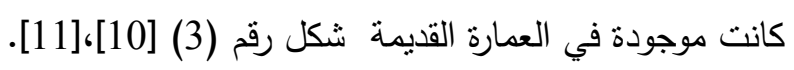

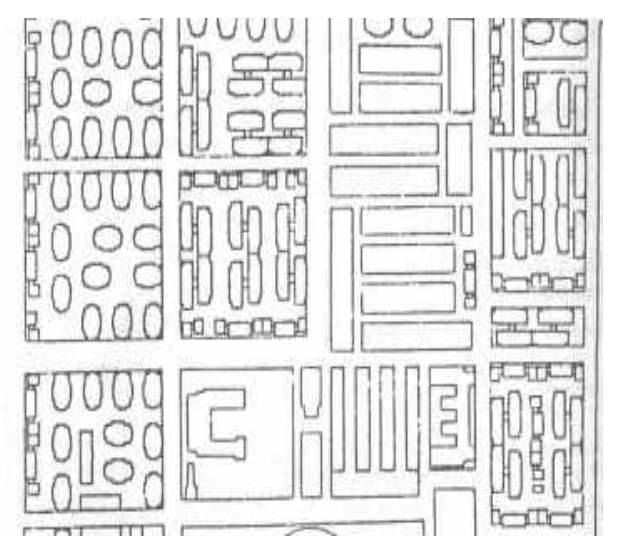

شكل رقم (3) النسيج

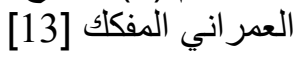

\section{5- القيم في العمارة المعاصرة في مدينة اسيوط كمثال.}

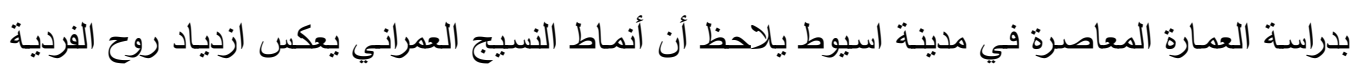

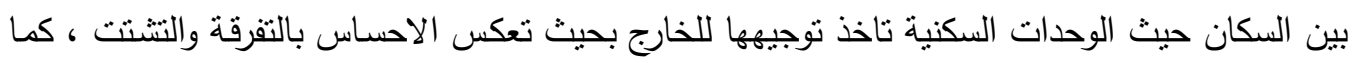

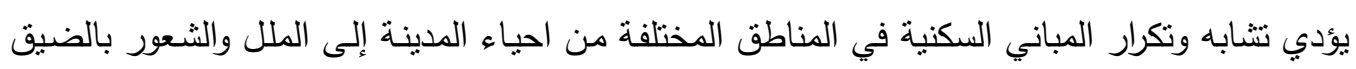




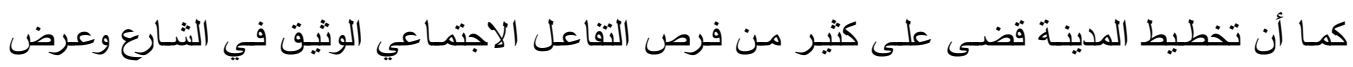

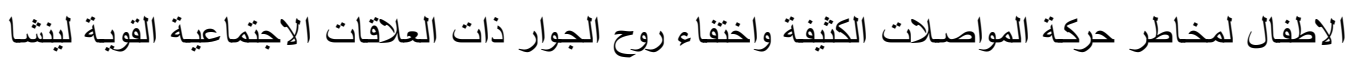

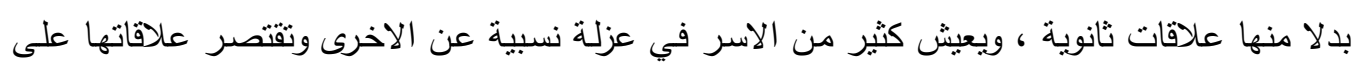
العلاقات العابرة.

ومن خـلال دراسـة العمارة المعاصـرة في مدينـة اسيوط امكن تقسيمها إلى ثـلاث انماط : نمط العمارة

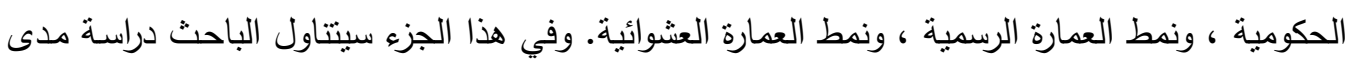
توفر القيم في تلك الانماط .

\section{4-1-4 القيم في نمط العمارة الحكومية.}

أولت الحكومة اهتمامها بتوفير السكن لهحدودي الدخل وكان ذلك بانثاء مساكن سريعة التصميم والتتفيذ

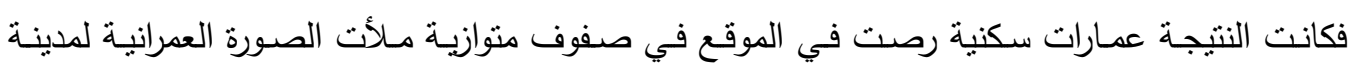

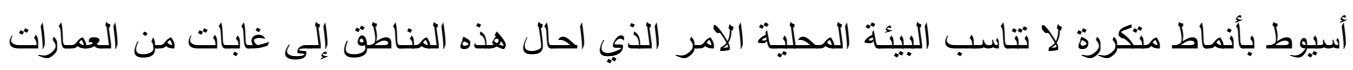

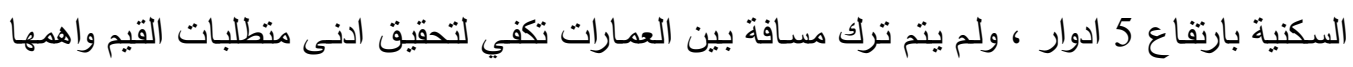
تحقيق الخصوصية وقد اكدت الدراسات ذلك حيث اشـارت إلى أن 80\% من المساكن تعاني من فقدان الخصوصية [12] شكل رقم (4).
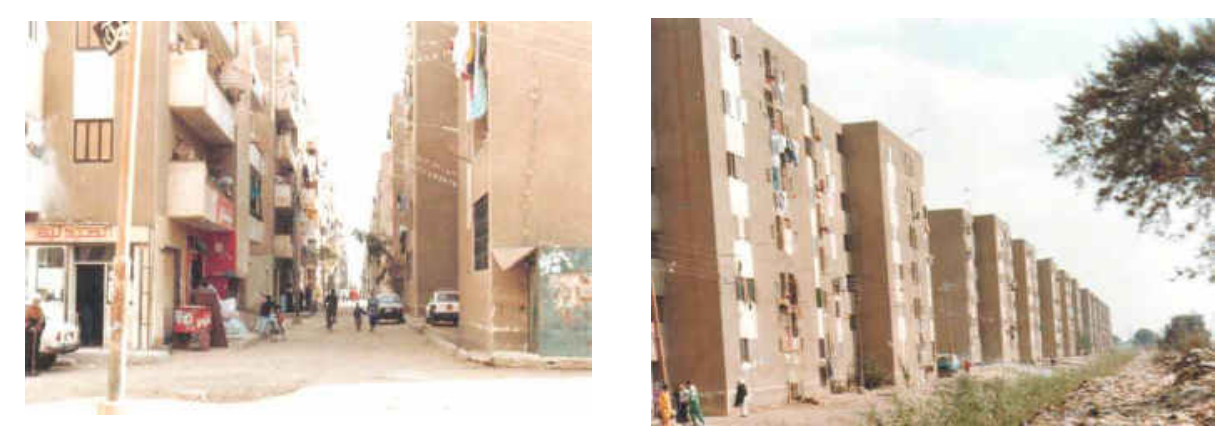

شكل رقم (4) مساكن حكومية بموقع إسكان حي الأربعين بمدينة أسيوط [14]

وقد أثنارت دراسة أخرى تمت على موقع اسكان حكومي ضخم بالمدينة يضم اكثر من 5658 وحدة

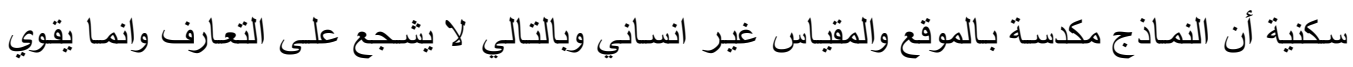

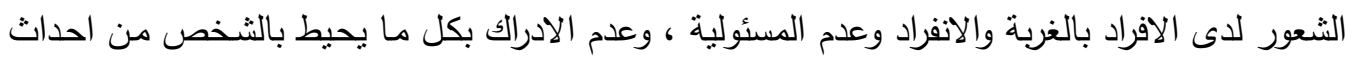

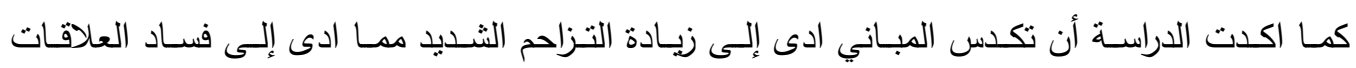

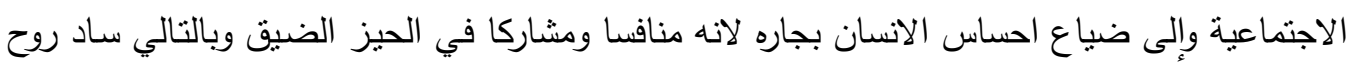


الصـراع بين الجيران وضياع حق الجار والاحسـاس بالالفة اضـافتا إلى فقدان الخصوصية الخارجيـة

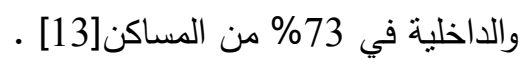

4-2-4 القيم في نمط العمارة الرسمية3

هذا النمط يعكس نمط العمارة العالمية ولكن يحكمه قانون المباني ويظهر هذا النمط بوضوح في ما يسمى ابراج التمليك وقد ادى ظهور هذا النمط إلى تمادي المستثرين بالتعدي على قوانين المباني الخاصة بالارتفاعات والبروزات لان الهدف الاساسي هو تحقيق اكبر عائد لاستثماراتهم. ومن خـلال دراسـة نمط العمارة الرسمية وجد أنها لا تحقق القيم المطلوب توافرها لما يأتي [9] شكل رقم(5):-

- - - المباني موجه للخارج وعروض الثوارع لا يحقق الخصوصية - ارتفاع بعض المبانى وصل إلى 36متر والمسافة المنروكة بينها من 6- 6-8متر . - - - ابعاد المناور غبر كافية لتحقيق الخصوصية. - الحوائط المستخدمة سمك 12 سم ولذلك لادئ تحقق الخصوصية السمعية.
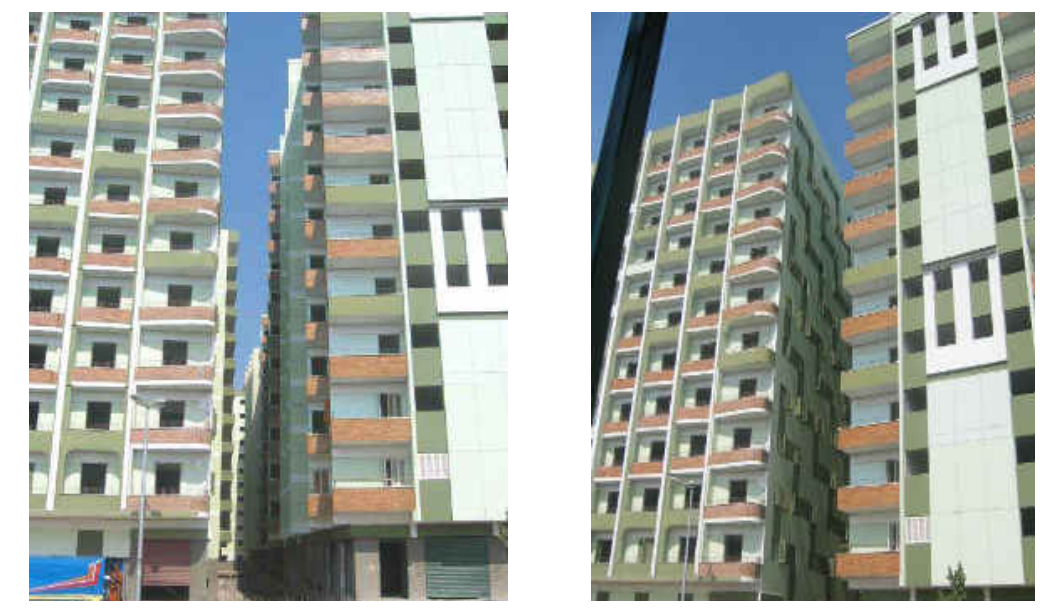

شكل رقم (5) تقارب المباني مع زيادة ارتفاعها إلى 36مثر أدى إلى القضاء على إلى

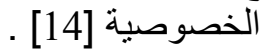

\section{4-3-4 القيم في نمط العمارة العشوائية.}

يتسم نمط هذه العمارة بأنها ليست لها طابع محدد فهي تقتح على الخارج على شوارع ضيقة ومتعرجة

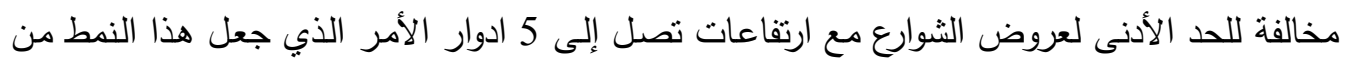


العمارة خـالي مـن أي قيم ، كما تتـاخل الأنشطة التجاريـة والصناعية فيها فتسبب ضوضـاء شديدة

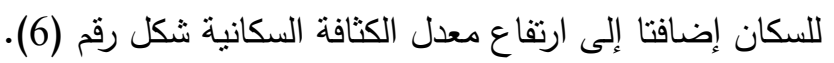

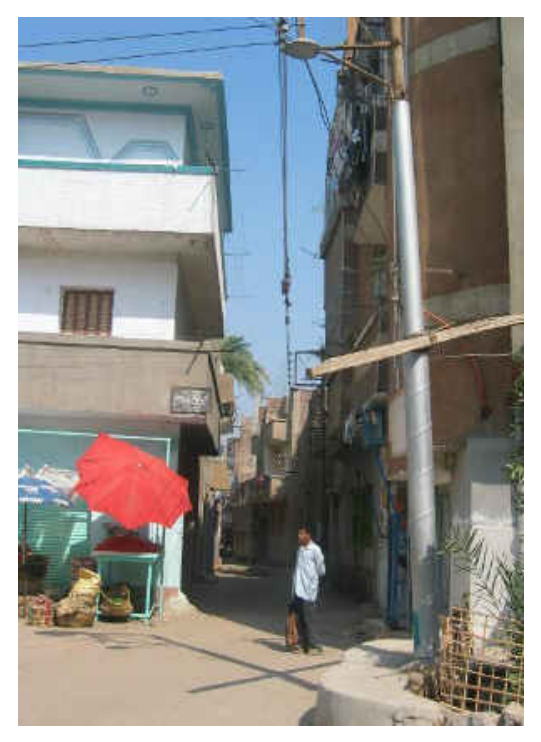

شـكل رقتم (6) مسـاكن عشـوائية تطل على شو ارع ضيقة [14 من من

\section{5- الخلاصسة}

خلص البحث إلى ان القيم المطلوب توافرها في العمارة تمثلت في تحقيق الخصوصية بأنواعها،

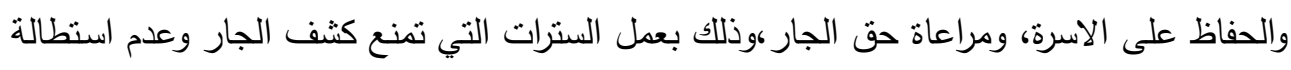

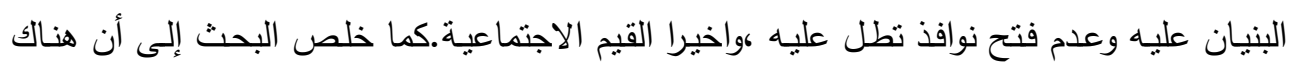

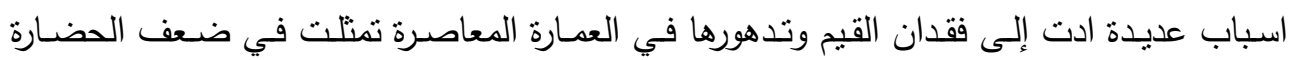

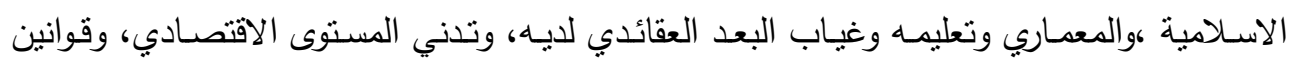

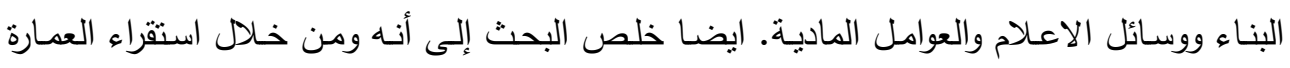

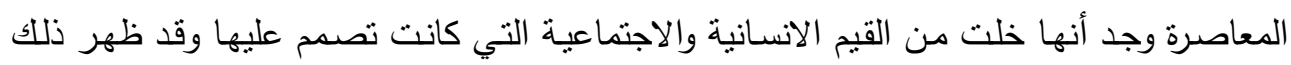

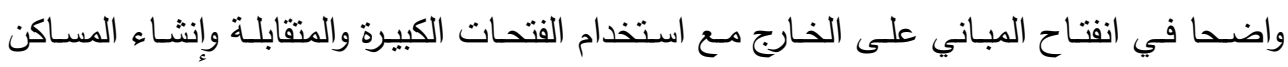

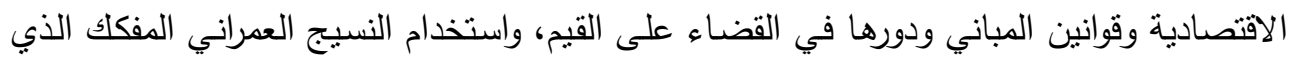
ادى إلى العزلة الاجتماعية وضعف الروابط بين السكان. ومن خلال دراسة الحالة التي تتاولها البحث خلص إلى ألى العماعلى العمارة المعاصرة في مدينة أسيوط خلت من القيم بل يمكن القول بانه لا يوجد قيم سواء كانت في العمارة الحكومية أو الرسمية والعشوائية.

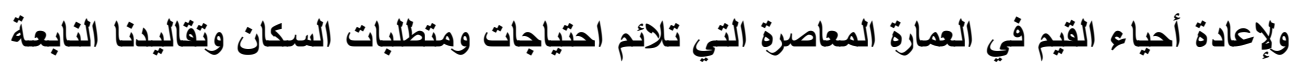
من عقيتتنا الاسلامية فانه يلزم اتباع التوصيات الآتية: 
* ضـرورة إعادة النظر في قوانين المباني بحيث يجب أن تراعي تحقيق الخصوصية بكل انواعها ومراعاة حق الجار والمحافظة عليه. * - الاهتمام بالعمق العقائدي لدى المعماري بحيث تعطى القيم اهمية كبيرة في اسلوب تعليمه.

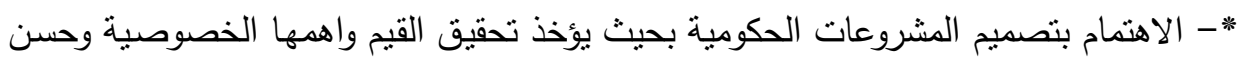

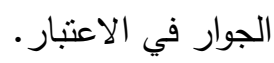

* - الاهتمام بدور وسائل الاعلام في اعادة احياء القيم وضرورة المحافظة عليها بين الناس.

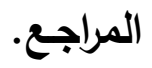

1- محمــ خيـر الـدين الرفـاعي. نحسو عمـارة اصـيلة ومعاصـرة تسـتمد معطياتها مـن القيم

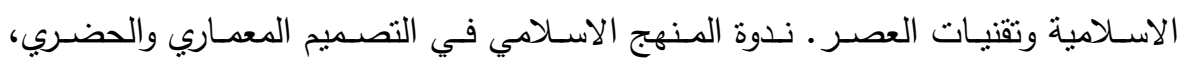

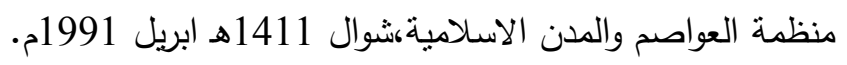

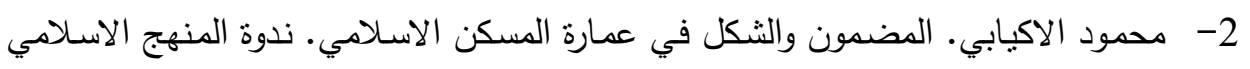
في التصميم المعماري والحضري، منظمة العواصم والمدن الاسلامية، شوال 1411هـ البرديل 1991

3- عبدالقادر حمزه كوشك. المنهج الاسـامي في تصميم العمارة. ندوة المنهج الاسـامي في

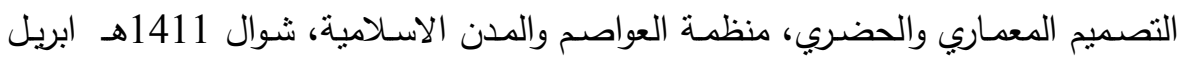
.1991

4- محمود احمد عبداللطيف. سبل تطبيق مفاهيم المنهج الاسـلامي على العمارة المصرية

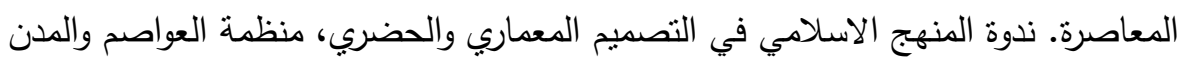
الاسلامية، شوال 1411هـ ابريل 1991م.

5- اسامة احمد مسعود. تاصيل القيم الحضارية والتراثية لبناء المدن في المجتمعات الاسلامية

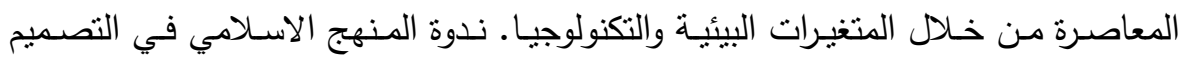

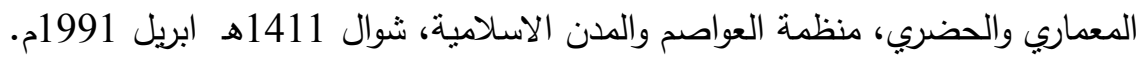
6- احمد هلال محمد. نمط البناء الافقي الموجه إلى الدلاخل النموذج الامتل لعمارة الصحراء.

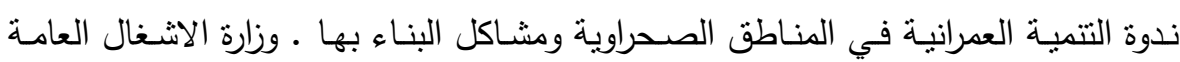

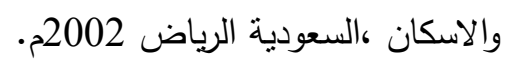
7- عزة حسين فؤاد. تأصيل القيم المعماريـة الاسـلامية في العمارة المصرية المعاصرة. ندوة المنهج الاسـلامي في التصميم المعماري والحضري، منظمـة العواصم والمدن الاسـلامية، شوال 1411هـ ابريل 1991م. 
8- ناصر بسيوني عبدالعزيز ـ تأصيل قيم الجوار في العمارة السكنية المعاصرة في مصر .

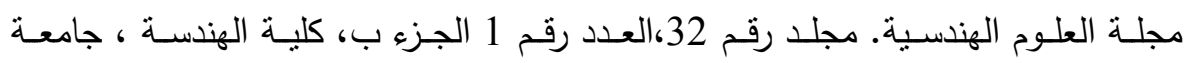

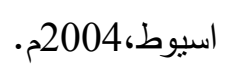

9- احمد هلال محمد. مفهوم الخصوصية في عمارة المدن المصرية المعاصرة(حالة دراسية

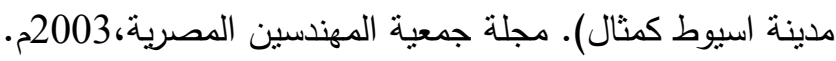

10- حسن عوض بشارة. القيم الاسلامية والتراث المعماري بالمدن الاسلامية. مجلة عالم البناء، العدد 90، ابريل 1988م.

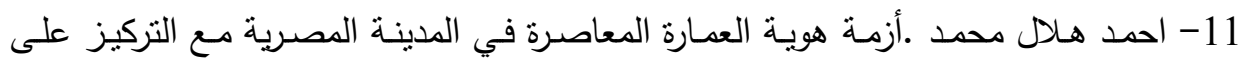

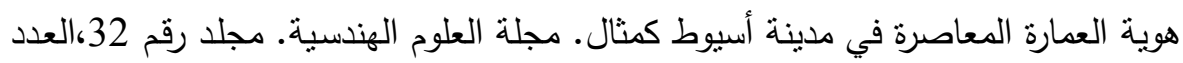

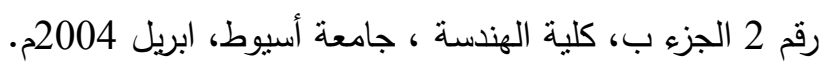

12- احمد هلال محمد. تأثثر التغيرات السكانية على التصميم والتخطيط البيئي في المدينة المصرية المعاصرة. المؤتمر الحادي عشر لمنظمة المدن العربية ، المعهد العربي لإنماء المدن، تونس، 26-28 مايو 1997م.

13- احمد هلال محمد ـ تقييم تجربة الحكومة للتمية في مجال إسكان ذوي الدخول المحدود في مدينة أسيوط- حي مبارك كمثال-. مجلة جمعية المهندسين المصرية. 14- صور نم تصويرها بواسطة الباحث وزميله د. ربيع محمد رفعت بمدينة أسيوط دئه 